\section{orignTat COMNTUNICATIONS.}

\section{CLINICAL NOTES ON CHOLERA.}

By W. LAUDER LINDSAY, M.D., late Resident Physician of the Surgeon Square Cholera Hospital, Edinburgh.

\section{[Concluded from page 971.]}

TATURAT INFLUEXCE OF CHOLERA ON PLANTS AND THE LOWBR ANIMAXS.

Tris subject appears never, in this country especially, to have attracted that amount of scientific attention which its important bearing, not only on the natural history of cholera, but of epidemic diseases in general, unquestionably chants and demands. In various parts of the continent and India, and to a slight extent in Scotland, observations on cholera-epizootics and on the diseases of the lower, particularly the more familiar domestic animals, during epidemics of cholera, have occasionally been recorded: but this may be said to comprise the bulk of our extant literature on the subject. Notwithstanding that thousands have had daily opportunities during the present and former epidemics, in country districts-where observations of this kind may be easily made-it is remarkable that few or no facts bearing on this point have been recorded. It is matter of regret that, while a countless amount of labour has been expended in repeatedly going over ground that has been trodden till it is almost barren, in recording mere symptoms or the results of empiric treatment, a boundless field for original research, which promises to be productive of the most valuable results, lies undisturbed before us, or only trenched upon by a few of the more bold and enterprising pioneers of medical science. Many observers in this country deny or disbelieve that the lower animals are subject to cholera, or to any disease having a semblance thereto, maintaining, on the contrary, that cholera is peculiar to man; many more hold in derision the opinion that cholera is transmissible between different species and geners of animals; and very few indeed will be found ready to believe that cholera is propagable from man to the domestic animals, and vice vers $d$, or that such a phenomenon as the transmissibility of disease from man to the brutes is within the bounds of possibility. Though we possess little real in. formation on these points, we have accumulated sufficient data to convince us that these phenomena are not mere speculations or theories, but can be proved to be facts. It is the object of my present paper to point out to observers that this subject and its congeners constitute a wide and important gap in the study of the natural history of cholera, which must be filled up before we can expect to make advances in our knowledge of the intimate nature of this disease ; to indicate their important connexion with the study of the laws of epidemics and with medical science in general; and to mention shortly, in detail, the heads of the inquiry which appear to me more immediately to invite research and eluciảation. Observations ought to be carefully made and accurately noted, as nearly as possible simultaneously, and under similar circumstances, all over the world: the general deductions founded on such an accumulated mass of data could not fail to possess the highest scientific value. Many branches of the subject could be followed out by persons possessed of little or no medical or scientific knowledge: others naturally fall within the province of the veterinary practitioner; but it would be advisable to have the investigations carried out, or at least superintended, by men eminent in science, fully impressed with the important bearings of different points of their researches on general science, and fully capable of appreciating all the data calculated to elucidate the special question at issue, as well as to illustrate general medicine. Experiments, analytical and synthetical, promise to be of sp scial service in an investigation of such a nature. I pould humbly suggest this as a worthy subject of inquiry for the medical societies of our country $\rightarrow$ more particularly such societies as the Apidemiolojical-whose members are scattered orer the world, and who could contribute, at a trifling expenditure of time, towards the collection of a vast mass of valuable data, which might in due time be arranged and classified.

In connexion with the effects of atmospheric contamination by the choler poison on the production of disease in plants and the lower animals, more particularly those which are cultivated or domesticated, it appears to mo that the following points are specially worthy of research, bringing to bear thereon all the light of modern histology, chemistry, pathology, etc.

I. The Epidexic Diseages or Phants, especially those extensively cultivated by man. Their etiology, pathologJ, prevention, and cure.

A. Predisposition to disease in plants.

1. Influence of meteorological conditions or changes.

a. Especially with reference to electric and hygrometric state;

b. Presence of miasms or chemical poisons ;

c. Deficiency or excess of heat, light, etc.

2. Geological structure and physical configuration of the country.

3. Presence of poisonous matters in the soil, etc.

B. Transmissibility of disease, by contact, among different individuals, species, and genera.

$C$. The general laws of epidemics in plants.

II. EPIzOOTIC Diseases of THE LOWRB Amixars, paricularly domeaticated species.

$A$. Causation; proximate and remote.

$B$. Predisposition.

1. Atmospheric conditions and changes.

2. Individual structure, constitution and idiosyncrasies.

3. Domesticity or civilisation.

a. Errors in food.

b. Exercise.

c. Cleanliness.

d. Ochlesis and its effects.

4. Geological structure and physical configuration of country, etc.

$C$. Mode of origin and propagation.

$D$. Connexion with the diffusion of man and his condition of civilisation.

$E$. Contagiousness.

1. Transmissibility, by contact, between different species and genera.

2. Transmissibility, by contact, to man, and vioe versa.

F. Prevention and cure.

$G$. Species and genera most liable to be affected by particular diseases. Causes of such susceptibility.

$H$. Difference of type and degree of the same, or different diseases in the same or different animals. Causes productive thereof.

I. Study of diarrhoid, dysenteroid, or choleroid epizootics, especially in animals riearly allied to man in structure and habits.

1. Transmissibility. Modifications in type therefrom resulting.

2. Mode of death.

3. Pathology of intestinal lesions.

$K$. Study of epizootic cholera, especially in the dog.

1. Predisposition.

2. Transmissibility, etc., as above.

3. Symptomatology.

a. Essential symptoms.

b. Accidental or accessory symptoms.

4. Experimentation on artificial propagability.

a. Between different individuals, species, and genera.

b. Between different individuals, species and genera, and man, and vice verad. Differences in types thereby produced.

c. Effects of hygienic and remedial means in,

1. Prevention.

2. Cure. 
d. Hor far is the dicense moditied, increased, or diminished in power by transmission. Laws of such modifications.

e. Circumstances farourable and unfarourable to contagiousness or transmissibility.

$\boldsymbol{I}$. Iiability of various species and geners to diseases, marked by one or more of the following prominent symptoms :-

1. Diarrhoen, especially serous, mucoid, or lightcoloured, and fluid.

2. Vomiting, especially abilious.

3. Cramps, unconnected with convulsive disease.

4. Collapse, having no apparent or proportionate causc.

5. Unusual thirst, without cause.

M. Liability of various species and genera to intestinal diseases, or to diseases marked by intestinal lesions. Symptomatology, pathology, etc., of such diseases.

$N$. Liability to sudden death during epizootics. Pathology of such cases.

0. The general laws of epizootics.

III. The Motuat Relations of Epidemics, occurring simultaneously or successively, in plants, the lower animals, and man.

A. How far are these, though differing in characters, due to the same atmospheric poison?

$B$. Within what limits may the same aerial poison be held to cause different effects in these classes of organised beings, according to different external circumstances?

$C$. To what extent do different atmospheric poisons or different states of the same poison affect the same animal or plant under different circumstances ?

IV. Sporadic Diseases of the Lower Axixals, common or peculiar to different species and genera.

$A$. Anatomy, healthy and morbid, of the digestive apparatus, especially of domestic animals, and such as most closely resemble man in structure.

1. How far admitting of the exhibition of certain physiological phenomena, e.g., vomiting ?

$B$. Comparative susceptibility of different species and genera to be affected by the same disease.

$C$. To what diseases or classes of disease are particular species and genera liable?

$D$. Comparative liability of certain species and genera to exhibit certain symptoms.

$E$. Liability to sudden death.

1. Pathology of such cases.

$F$. Circumstances producing modification in type.

1. Anatomical or physiological structure or peculiarities.

2. Domesticity, and habits resulting therefrom.

$G$. Sporadic cholera, and the diseases with which it may be confounded. Diarrhoea, dysentery and intestinal affections.

1. Predisposition.

2. Transmissibility, etc., as above.

Epidexics amora Phants. It has frequently been noticed that, prior to the outburst of cholera in a district or country, there have existed extensive and serious epidemics among various plants, widely cultivated by man on account of their economic uses, e. g., the potato and vine. Many, perhaps mistaking a merely coincident for a causal relation, have at once assumed that these diseases were produced by the same cholera poison, and might be considered as cholera ; befieving that, while this poison is not sufficiently abundant or concentrated to produce disease in the lower animals or man, it is fatal to certain cultivated plants. In support of the latter opinion, the fact is adduced that the disease of the potato-the regetable most frequently attacked in such circumstances-is usually considerably prior in date to epizootics among the domestic animals, or to cholera in man. This also may be a mere coincidence $:$ it is possible that the epidemic may, and does, happen equally during and subsequent to general outbreaks of cholera in man. We ars not in possession of sufficient data to warrant us in believing that the potato disease, or vine blight, at all times, or under certain circumstances, is allied or equivalent to eholers, or is eren produced by the cholere poisgn in tho atmosphere. But it must be confessed that our knowledge of the sporadic and epidemic diseases of plants is still rery meagre and unsatisfactory : much research is necessary ere the subject be placed on a good scientific basis. It is, however, abundantly ascertained that many plants, especially cultivated ones, are liable to disease from the following, which may be either predisposing or exciting causes :

I. By certain alterations of the ordinary conditions of life, e. q., excess or deficiency of light, heat, moisture ; certain chemical compounds in the soil ; electric changes in the atmosphere, etc.

II. By contamination of the air or soil, with miasmata, or poisonous gases.

III. By the growth of fungi and other parasites.

1v. By mechanical injuries, e. g., the bites of insects.

Experience informs us that of all the predisposing causes of diseases in plants, cultivation is the most powerfulthat is, the production of succulency or the increase or hypertrophy of the cellular tissue at the expense of the vascular, which is more permanent and durable, and less prone to decay and death. We know something, moreorer, of the results of an epidemic constitution of the atmosphere on the health of plants, but nothing of its cause, which is equally inscrutable and mysterious as the cause of cholera in the human subject. The potato disease has usually appeared simultaneously in different countries, or orer extensive districts of country ; the epidemics are gradually becoming less extensive, but more frequent; its cause appears to be $\bar{a}$ general atmospheric contamination, the precise nature of which our ordinary means of scientific research hare as yet failed in ascertaining : in these respects it resembles cholera. Many of the assigned causes are as probably $\epsilon$ ffects, e. g., fungi, and insects of various kinds. The discase is said to be contagious, the agent by which it is propagated heing supposed by many to be the minute and extremely light spores of the parasitic fungi which attack the plants. Some observers have succeeded in propagating the disease artificially, by mixing sporules of the fungi, taken from the leaves of diseased plants, with water, and applying it to the roots of healthy individuals. This fact is ingeniously taken advantage of by the supporters of the fungus theory of cholera, who can hereby extend their theory so as to include and account for the causation of epidemics in plants and the lower animals, as well as in man; but this mode of theorising has not advanced our knowledge of the nature of cholera in either of these classes of organised beings. The results of the poisonous influence of the epidemic miasma on the health of the potato are structural disorganisation, alteration of the cells and vessels and of their contents, proclivity to the growth of fungi; etc. It is admitted that predisposition has much to do with the attack; every plant is not attacked, but only such as, from a combination of several of the efficient causes which render plants subject to disease, are in a weakened or favourable condition.

Epizootics is Domesticated axd Wild Animals, and Diseases of THE Lower Avimals dering EPInemic Choners. A series of rerv interesting reports on the influence of epidemic constitution on animals during the prevalence of epidemic cholera in Lower Austria, Moravia, Galicia, and Bohemia, under the auspices of the Faculty of Medicine of Vienna, was laid before the Imperial Government in 1835.* The observers, by whom they were drawn up, found that the general health of all animals was affected in various ways; that, in many, especially of the domestic animals, the symptoms and pathology of the produced epizootic closely resembled those of cholera in man; that birds suffered in greatest numbers, and that the disease in them most nearly approximated the characters of human cholera; that dogs, cats, and havea also died in great numbers; and that, in domestia ... the epidemic influence of choloxold ath. un as their digeters of the epi-

Jahrb. des CEst. Staate3, 183.j, or Brit. and Foreign Medical Rerier. 
structurally resembled that of man: that, for instance, ruminants, which cannot, from the structure of their stomach, exhibit the phenomenon of vomiting, were comperatively exempt; and that aquatic animals, such as fish, arabs, and beches, also died in grest numbers. From the dats collected, it was concluded that the rarious forms of dat collected, it was concluded the observed in the various classes of animals were too intimately connected with the cholera opidemic to be merely coincident or due to sccidental causes; that cholera must in these districts have been pandemic; that the atmospheric poison was alike poisonous, therefore, to man and the lower animals ; that the poison could not have been confined to the atmosphere, seeing that aquatic animals were equally attacked by the same or similar disenses; and that it was most deleterious to birds, which are well known to be very readily affected by atmoepheric changes.

In different parts of India it has also been noticed that, coincident with cholera, cattle have died in equal or greater numbers than man; and in the same, and other countries, epizootic and sporadic cases of cholera, during epidemic cholera, have been observed in horses, dogs, cats, camels, gosts, geese, ducks, common fowls, monkeys, fish, the zebra, and racoon. In different countries, different species of animals seem to have been chiefly affected, e.g., the cholera in Dantzig, during its first spread over Europe, was preceded by a cauine epizootic.

Professor Dick, in a paper on Cholera in Domesticated Animals, ${ }^{*}$ describes a cholera-epizootic, chiefly affecting horses and cows during the first epidemic visitation of Edinburgh by cholera in 1831-2. In various of the pastoral districts of Scotland, the farmers have suffered severely and repeatedly from the sudden and extensive loss of valuable horses, cattle, sheep, pigs, and fowls, during, but more frequently prior to, epidemic cholera. At the same period, birds have been found to die in large numbers, more especially crows, rooks, blackbirds, and sparrows. The cause of the phenomena could not at the moment be satisfactorily explained; but, on the subsequent breaking out of cholera, they were immediately connected, whether correctly or not, as cause and effect. Under similar circumstances, game has been observed to perish to a great extent. During the present summer, in the Highlands of Scotland, there has been a very destructive epizootic among young grouse. "In Perthshire," says a newspaper at the time, "the young broods of grouse are suffering so much from a disease which is supposed to be tape-worm [?], that, in some districts, there will be a total failure of young birds." In many cases, the assigned cause of the epizootic is disease of the plants on which the respective animals chiefly feed; but, granting this to be the case, which, however, is merely an inference or theory, we are just brought back to the point whence we started, viz., the cause and nature of the epidemic in the particular plants.

Several cases are recorded of dogs and cats, which have accidentally licked the evacuations of cholera patients, being, at varying intervals thereafter, seized with and dying of the disease; and more than one case is extant where a dog thus seized communicated the disease to its mistress, in whom it proved rapidly fatal. If such cases be accepted as authentic, they are valuable specimens of the communicahility of the disease from the lower animals to man. Marshall, of London, mentions a very interesting case of a dog which swallowed some of its master's choleraic dejections, and which lay under his bed from 4 A.x. till 2 P.x., when he died of cholera: hereupon he smelt the corpse all over, and, ten hours after swallowing the evacuations, was himself seized, and subsequently died of the disease. The seizure in this case, though apparently attributable to the dejections, was more probably due to the effluvis from his master's body, bqth while alive and after death. In other cases of dogs and cats, which have been seized after licking the vomit or dejections of cholera patients, it also admits of considerable doubt whether the cause of this seizure was not the rolatile poison srining from thementions, or from the skin or lungs of patients, rather then the direct action of the eracustions on the mucous membrane of the intestinal canal. If, moreover, the expariments which I made last winter in Edinburgh should be repented and corroborated-as they may possibly soon be in France-it will be proved that the disease is also transmissible from man to the lower animals $\rightarrow$ point whose importance, if established, it is difficult at present to estimate. Multiplied observations and experiments in France prove that cholera is not only transmissible by contagion between different individuals of the same species, but between individuals of different species and geners. Instances are given of its communicability, not only from fowl to fowl, or from fowls to rabbits, and vice versd, but from geese, ducks, chickens, peacocks, guinea-fowls, and rabbits, to goats, horses, and dogs.*

In some of the instances abore quoted or referred to, no information is given of the precise nature of the disesse so. extensively and rapidly fatal to the animals; but, from its connexion, with regard to time, with epidemic cholera, it is presumed to have been choleroid; in others, a consideration of the symptoms leads to the supposition that the epizootic was choleraic; while, in the remainder, the symptoms and pathology leave no doubt in the minds of the observers as. to the choleraic nature of the disease. But the great evil and defect of the majority of observations hitherto recorded on this subject is the want of accurate pathological information. Without a detailed and careful account of the pathological conditions, as well as the description of the mere symptoms, which are exceedingly apt to lead into error, few cases can be received as genuine or safe data on which to. found general deductions or laws.

In debating the question, whether a given disease, sporadic or epizootic, in certain of the lower animals, is cholera or not, several points demand our careful consideration. In hazarding an opinion on a subject of such acknowledged difficulty, it is frequently necessary to take into consideration not only the symptoms, mode of death, and post mortem appearances, but all the special and collateral circumstances of the attack and death, before a safe judgment can be arrived at.

Observation of the symptoms alone is calculated to lead into error. In several comparatively insignificant affections of our lower animals, some of the symptoms usually considered characteristic of cholera in man are frequently exhibited. For example, vomiting is easily induced in the dog and cat; voluntarily so in the latter. Diarrhcea in these animals is a common result of unusual or bad food. Bilious romiting and purging sometimes coexist, and several animals are liable to fits resembling cramps. Again, many animals are incapable, from the structure of their digestive organs, of exhibiting certain classes of symptoms; e. g., ruminants cannot vomit. Sudden deaths are comparatively cominon among many of the lower animals, especially during epizootics. In some cases, a connexion has been traced, by pathological examination, with intestinal lesions; but, in the majority of cases, no endeavour is made to discover the cause of death, or it is attributed to the prevailing epidemic poison. In such circumstances, the necessity of post mortem examinations is too obvious to require comment. Professor Dick, of the Edinburgh Veterinary College, states that sheep, cattle, and horses, are often suddenly cut off, after a few hours' illness; but that the liability to sudden death is much less in dogs, though they are subject to a much greater variety of intestinal disorders. This liability is markedly different in herbivorous, carnivorous, and omnivorous animals. The same observer believes the fatal lesion in these cases to be acute inflammation of the intestines, and the cause of variety in liability he finds in the different nature of the food. The habitual use of excess of vegetable food is found to be a porerful predisposing cause of intestinal disease in the lower animals: hence the reason why the herbivora are so frequently attacked by suddenly

- Gazotte Hebdomadairo de Yedopine ef do Chirurgie, Oet, 13th, 1854. 
fital sonto inteatinal inflammations. It is noterorthy, in cemexion with this observation, that the cholera seizure in the human subject very frequently follows, more or less inmediately, a large or unusual meal of regetable food: and the firat romits of cholera patients usually consist of undigested food, especially of a vegetable nature. I have ropeatedly had occasion to observe this in regard to potatoes and broth. The poor, who are the principal victims of cholern, are compelled to live greatly on regetable food, on account of its superior cheapness, and its being more readily obtained. They are also in the habit, for similar reasons, of habitually using the same kind of food; many making the basis of three meals a day potatoes, porridge, or tea and bread. In the majority of cholera cases which have fallen under my own observation, there was a habitual deficiency, for long periods previously, of animal food. It appears that there is an analogy between the symptoms of cholera in man and those characteristic of intestinal infiammation in herbivorous animals ; but it is obvious, from what I have already stated, that on mere similarity in appearances no definite conclusions can be founded. Blaine mentions that, in some kinds of diarrhoea in dogs, a glairy, frothy, mucous matter is discharged. This might be confounded with choleraic dejections. Obstinate diarrhoea in these animals frequently passes into dysentery, and the rectum becomes inflamed and ulcerated: here we should have a pathological feature resembling lesions frequently met with in human cholera. In such cases, incessant thirst is a constant symptom. Dogs are, moreover, subject to cramps in the muscles of the limbs. Many diseases of the dog, therefore, accompanied by cramps, vomiting, and purging, or one or more of these symptoms, might, especially during an epizootic, and while epidemic cholera was raging in man, be mistaken for cholera, unless we are very guarded and accurate in our observations. I cannot help thinking that such mistakes have occasionally been made in describing as genuine cholera diseases in the lower animals which merely presented analogies or resemblances. This tendency to jumping at conclusions-this lcoseness in descriptire languagecannot fail to be dangerous to the interests of true science. If this opinion be correct, we must deduct a considerable number from the list of instances on record of cholera, sporadic and epizootic, among the lower animals: at the same time, I beliere that, when the subject of their susceptibility to diseases caused by the cholera poison is investigated in the way I have indicated, we shall find that cholera is far from being essentially human.

$\Lambda s$ in man, the subject of predisposition, in relation to the effects of the cholera or other atmospheric poisons on the health of animals, is of great importance. The effects of food, hahits, atmospheric vicissitudes, etc., in predisposing to other epizootic diseases, are sufficiently acknowledged in veterinary medicine. We cannot expect the predisposing causes to be the same as in man: we must make certain allowances for differences arising from their position in the zoological scale; varieties in anatomical structure; physiological peculiarities; differences in habit, and exposure to external circumstances, etc.: and we must expect differences in the type of diseases resulting therefrom. Blaine mentions that deficient exercise is extremely detrimental to dogs: it is, he says, "the cause of nearly one-half the diseases of dogs". It tells much more quickly and screrely on the canine than on the human species; the life of the dog being one of constant activity. The common consequences of confinement in him are fits, mange, canker, asthma, obesity, and dropsy. In experimenting on the communicability of cholera in the lower animals, it is important to bear in mind that confinement, with limited exercise, assisted by the habitual use of food bad in quantity or quality, and by the respiration of an atmosphere contaminated by noxious effluvia, is apt to induce a vitiated state of the general health, marked by cutaneous eruptions, or by other evidences of derangement of the blood. In a dog, one of the subjects of my own experiments, neither diarrhoa nor cholera was induced by the means employed, but mange. It was confined with a number of other ani- mals, which were fed on the evacuations of cholera patients; it partook very sparingly of the viands supplied, but seemed much affected by the confinement and by the efin via arising from the food, as well as from the evacuations and bodies of its companions. Blaine also mentions that dogs, especially such as are much domesticated, are liable to be affected even by slight atmospheric changes. As in man, over and above the ordinary predisposing causes of disease, we must expect to find the cholera poison operative in some, and inoperative in other animals. The cause of this phenomenon has usually been ascribed to constitutional peculiarities or idiosyncrasies belonging to the individual.

As bearing on the question of the contagiousness or transmissibility of cholera in animals, it is well known that in several diseases of the $\mathrm{dog}$, which are contagious, the effluvia from the bodies and eracuations of affected animals are highly dangerous to healthy individuals, and are apparently the most efficient agents in communicating disease.

It is useful to bear in mind that cpizootics are common enough at times and places when and where cholera does not exist; where, therefore, it is presumed that the poison producing cholera is not present in the atmosphere, and there can be no causal relation between them; and sudden deaths in various species, arising from the same visceral lesions, are equally common during these periods. But the mere fact of epidemics in plants or epizootics occurring during, or shortly prior to, epidemic cholera in man, is far from being sufficient proof, even presumptive, that these also arc essentially choleraic in their nature. It answers very well in theory to suppose that plants, the lower animals, and man, are attacked in the orler in which they have been mentioned by the cholera poison, because analogy would lead us to infer that, while a sniall amount of a volatile poison diffused in the atmosphere may be fatal to plants, especially such as are predisposed to disease by cultivation, it must accumulate to a large amount before it is sufficiently concentrated and powerful to destroy life in animals or in man. I hope, however, I have said enough to prove that, in the examination of this subject, theory must be laid aside for fuct; otherwise mere coincidences will be again and again mistaken for connexion, and serious errors may arise.

It may not be an inappropriate conclusion to these remarks to add a few words on

Experimentation on the Traxsuissibility of Cholera between different individuals, species, and genera of the lower animals, and more particularly between them and man. Like the study of the naturul influence of cholera on animals, this subject, while it appears to have attracted considerable attention among continental observers during former epidemies, has never been investigated in this country with the care or to the extent its importance demands. Accordingly, while we find in Berlin, Venice, Milan, Warsaw, Dorpat, Greifswald, and other continental cities, such men as Schmidt, Eichstedt, Meyer, Naunias, Calderini, Novati, Bossani, Freschi, Scmmola, and others, investigating the subject with svirit, in Britain only one observer, Marshall. of London, secuns to have devoted any attention thereto. His paper, however, is one of great interest, not only from the detail of personal experiments, but from the abstract which it gives of the most important investigations that had been previously made on the continent.*

The morlus operanili of the earlier cxperiments has been-

I. Inoculation of the cellular tissue, chietly of the thigh.

II. Injections into-

a. The reins, particularly the jugular.

b. Cellular tissue.

c. Stomach.

d. Mouth.

e. Rectum.

And, in the more modern experiments- 
II. Inhalation of the effluris from the clothes of choler patients; and

IV. Causing the animals to swallow, voluntarily, fluids and solids of various kinds.

The substances used as the agents of experiments have chiefly been the

I. Human blood-

a. Drawn from the veins of cholers patients in different stages of the disease, but especially during collapse.

1. Pure, or mixed with water, or peroxide of iron, common salt, and other chemical substances.

2. Fluid, or the congula.

3. Cold, or heated to various temperatures.

4. Defibrinated or not.

5. Fresh, or after being kept for various lengths of time.

b. Taken from the heart, great vessels, liver, or other viscera, after death, in different stages of the disease.

II. Blood of animals dying or dead of cholera artificially induced, or dying of the results of experiment, whether by purulent infection, irritant poisoning, air in the reins, sequelæe of the mechanical wounds, or by other means.

IIr. Dejections of human cholera patients, collected during various stages of the disease, but more particularly during collapse.

a. Crude, or

b. Filtered, and flocculi or salts remored.

Iv. Clothing saturated with the

\section{a. Sweat,}

b. Breath, and

c. Dejections of patients, also labouring under different stages of cholera.

The animals which have mostly been the subjects of experiment have been rabbits, guinea-pigs, goats, fowls, dogs, and cats. I am not aware whether the horse, cattle, sheep, or animals similar in structure, size, and habits, have been experimented on. I cannot do more at present than briefly recapitulate, by way of illustration, the results arrived at by some of the observers whose names have just been mentioned.

Meyer, of Berlin, inoculated dogs with the blood and evacuations of cholera patients, with the result of producing symptoms identical with those of human cholera; viz., unequirocal rice-water stools, blueness, coldness, cramps, a tarry condition of the blood, and non-secretion of urine.*

Namias of Venice inserted blood, drawn in the collapse stage, into the cellular tissue of the thighs of rabbits. Of nine cases, all died within periods varying from twenty-four hours to eight days. In most of them, a whitish glutinous matter was found on the floor: in some, there was ecchymosis of the skin ; in others, the wound was found suppurating, the bladder was full, the fæces were merely unusually fluid, and there was no alteration of the viscera. The blood of animals, which died under or from the effects of the experiments, was employed in the same way, and with similar results. In one of the cases, the bladder was empty, and the skin was covered with brown spots, resembling ecchymoses. He found that the earlier in collapse the blood was drawn, the more rapidly fatal were its effects when inoculated. $\dagger$

His experiments were repented by Calderini of Milan, with nearly similar results. Bossani, Freschi, and Novati of Bergamo, and Semmola of Naples, met with negative effects, operating on rabbits, guinea pigs, and dogs. A commission of inquiry was appointed by the Royal Academy of Sciences, to repeat, and report upon Semmola's experiments on cleven animals of the species just mentioned. The result3 werc also negative. Novati repeated his own experiments, on a much larger scale; and satisfied himself that the transmission of the poison through the bodies of animals does not weaken its power, and that its effects are

- Virchow's Archives of Pathol. Aust ; or Ann. der Charité. Third yenr.

4 Bulletino delle Scienze Mediche, Sept. 1t3;; r. Brit. and toreign Med.-Chir. heriew, January IE38. much more powerful when communicated to individual of the same species, than to those of different species and genera. To specify some of his experiments: In two rabbits, inoculated with blood from living cholern patients, no effect was produced. Of twenty-one rabbits, inoculated with dead cholers blood, eleven died between the third and seventh day; in two of them, the blood used had been that of a foetus whose mother had cholera : most of the survivors showed choleraic symptoms. Of twenty-one rabbits, inoculated with the blood of a rabbit that died of cholers, nineteen died between the first and fourth day; and two others had choleraic symptoms. Living blood was injected into the mouth of one, and into the rectum of another rabbit: no effect followed. Living blood injected into the veins proved instantly fatal. The blood of the rabbit thus killed was injected into the veins of eight other animals: seven of them died very rapidly. One, whose femoral vein was tied, was, however, inoculated on the inside of the thigh, with the same blood, without injury. Dead blood injected into the reins proved fatal in thirty hours. Fluid dejections of cholera patients, inoculated with a vaccination needle, produced no effect. Those of a rabbit labouring under the disease, inserted into incisions in the skin of three animals, killed two of them, one in twelve hours, and the other on the third day; the third animal barely escaping with its life. A large rabbit was enveloped in a shirt imbued with the perspiration of a cholers patient: the only effect produced was extreme prostration. Remembering the similarity in effects between poisoning by cholera and by arsenic, and judging that the antidote to the one might also prove a protection against the other, he mixed cholera blood with peroxide of iron (ferrugo), and injected into the reins. The experiment was fatal in nineteen hours. Blood mixed with common salt proved fatal in sixteen hours.* Other continental observers have mentioned that clots of dead cholera blood, placed under the cellular tissues, or injected, were productive of negative effects.

Marshall, of London, not only repeated many of the experiments of continental observers; but he varied them in accordance with improved views in zo0-chemistry, pathology, histology, etc., arising from modern discoveries in science. Thus, guided by the now well known fact that the introduction of pure blood of one species into the system of another produces, in the majority of cases, serious, if not fatal consequences, the cause of which phenomenon is mainly attributable to the fibrin, in his experiments he used. defibrinated blood. In some experiments, he mixed the blood with distilled water; in others, he filtered the dejections, and deprived them of their salts and flocculi : in all the cases, he was probably careful as to the temperature, specific gravity, and freedom from impurities of the fluids employed in injection. Of nine cases, where he used the ejections or dejections of patients, three were fatal, with symptoms and pathological lesions in many points resembling those of cholera in man. Positive results were obtained only where large quantities of the evacuations were employed. He did not find that the removal of the flocculi or salts modified their effects. Negative results were obtained from injection of the jugular vein with dead blood, defibrinated and mixed with distilled water; and in other cases with fresh cholera blood. Inoculation experiments with the dejections were also negative in their effects in the hands of other observers.

As a contrast to the results above recorded, it is of some importance to notice a series of counter experiments, made by the same or different observers, tending to prove chiefly:

I. That the injection of putrid animal matter of any kind into the venous system of animals may be productive of fatal consequences.

II. That the symptoms preceding death, in many of these cases, are very similar to those of cholera.

III. That the introduction of the blood of a different species, or of pure blood not defibrinated,-and that various accidents or circumstances connected with the agent,

- Brit. and For. MLd.-Chirurg. Review, January $184 a$ 
mode, or subject of experiment, - may be followed by the reme results.

IV. That the injection or inoculation of cholera blood or eracuations, or the inhalation of cholera effluvia, under certain circumstances, produce negative, insignificant, or at least non-specific effects.

v. That the injection or inoculation of healthy human blood, or of blood taken from the human subject when labouring under various diseases, is followed by negatire results.

Namias obtained negative results, when he substituted healthy human blood for cholera blood, in inoculating rabbits. Novati wounded many rabbits sererely, and injected blood taken from men ill of various disorders, without bad effects.

Stich endeavours to prove, by a series of experiments, that many of the results above detailed, following injection into the veins, are solely due to the introduction into the system of putrid animal matter. He injected various putrid protein compounds into the blood or alimentary canal, having first carefully filtered them, so as to remove impurities. Death occurred in two to six hours, preceded by the following symptoms: Pulse and respiration became exceedingly weak ; extreme debility ; romiting ; dyspncea ; diarrhoea, the evacuations depositing a ricy matter: more rarely, convulsions and involuntary evacuations. After death, there was found lesion of the mucous membrane of the intestines, even though no symptoms had been exhibited during life: along with the spleen, kidneys, and abdominal glands, it was generally congested. When the putrid fluid was injected into the alimentary canal, the effects were similar, though much more slow in their development, and weak in intensity; they followed equally, whether cholera stools or ordinary fæces, which had been kept some time in water, were the agents employed.* Other experiments on the continent have abundantly proved that ten or twelve drops of " putrid animal matter injected into a dog's veins would cause prostration, excitement of the pulse, hard respiration, and a black or bilious vomiting". In my own experiments, the evacuations of cholera patients, used as food by dogs, appeared to produce diarrhcaa and other effects, in proportion to their putridity; and, from the general results, I was led to conclude that any similar putrid animal matter, under similar circumstances, would hare been followed by similar results.

It will be at once perceived, that between the results above detailed there exist very considerable discrepancies, which it is not easy to reconcile ; but, if the circumstances of the experiments be narrowly investigated, it will be found that, in the majority of cases, the results are either open to doubts as to their being choleraic; are negatire, inconclusive, and unsatisfactory ; or the modus operandi has been objectionable. The chief sources of fallacy in these experiments, and which it is often difficult, and sometimes impossible, to separate from the effects of the cholera poison, whether contained in the blood, evacuations, or fomites, are:

I. Accidents arising from the mechanical injury consequent upon injection and inoculation.
$a$. Entrance of air into the veins.
b. Pyæmia.
c. Phlebitis, etc.

II. Shock of operation, especially in timid animals, such as the rabbit.

III. Errors in the quantity or quality of the fluid injected or inoculated, especially with regard to
a. Chemical composition.
b. Temperature
c. Specific gravity.

Iv. Effects of introduction into the animal economy of blood of another species or genus.

v. Effects of introduction of pure blood, not defibrinated, even of the same species.

vI. Effects of introduction, especially into the renous system, of putrid animal matter in general. vir. Effects of introduction of putrid or irritant matters into alimentary canal.

We may also consider the following sources of failure, which are apt to occur in such investigations.

r. The non-production of a sufficient predisposition, by means which I have already detailed.*

II. The want of a sufficiently prolonged exposure to the exciting cause.

IIt. The circumstances of experiment not modified to make allowance for dissimilarity in structure and habits of the animals operated on.

rv. Inaccurate imitation of the whole conditions in which the human subject is placed prior to and during his seizure.

The objections first above mentioned do not hold good with regard to all the modes of experiment previously referred to. Inhalation, for instance, is undoubtedly one of the most powerful means of rapidly and thoroughly bringing the system under the influence of any volatile poison. The mode in which this has been applied has chiefly been to inclose the animal in a box along with some fomites for short periods, or to envelope it in the body clothes of patients, imbued with their perspiration or breath. It does not appear that the animals in any case were previously prepared to be brought rapidly or fully under the action of the poison, by the creation of an artificial predisposition. It is hoped that future observers will be guided by, and improve upon, the errors of omissions or commission of their predecessors. It is especially important to bear in mind the

I. Necessity for closely imitating the circumstances of the seizure in man, more particularly in regard to

a. Predisposition, produced by

1. Errors in diet.

2. Deficient exercise, confinement.

3. Ochlesis.

4. Respiration of a contaminated atmosphere.

5. Personal filth, etc.

II. Necessity of experimenting with the least possible risk of fallacy from accidents consequent upon the modus operandi, or the agents or subjects of operation.

IIr. Necessity of selecting, as subjects of experi:nent, animals as nearly as possible allied to man in structure and habits.

Iv. Necessity of fully producing an artificial cholera atmosphere.

v. Necessity of prolonged and sufficient exposure thereto.

It would be well, in future experiments, to vary as much as possible the media by which they are conducted. For example, as there is reason at present to beliere that the sweat and breath, as contained in fomites, are the most efficient agents in transmitting the disease, these might be separately collected and condensed, and applied in various ways to various animals. The air, saturated with effluvia from the evacuations, might also be collected and condensed, and the different constituents of the evacuations themselves could be separated and applied.

It is unnecessary here to recapitulate the reasons which lead me to attach a high valuc to this experimental branch of the natural history of cholera. It is matter of surprise that, amid the vast mass of cholera literature, this subject has been so long overlooked by British observers. None of the data hitherto advanced can yet be considered fully proved or established. The experiments made by myself, which are probably among the most recent, were published in a crude and unfinished state, merely with a view to stimulate others to prosecute an inquiry, which I had personally, to my great regret, no further opportunities of pursuing. They, therefore, require repetition and confirmation, before they can have a claim to be generally received by the profession as the exponents of certain laws in regard to the transmissibilty of cholera: or they must be proved erroneous by the combined evidence of several observers of acknowledged authority, before they can be set aside as

* Associatiox Jovasal, Sept. 15th, 1854; Edin. Med. and Surg. Journal, April and Oct. 1854. 
valueless. It is but proper that facts oppoced to existing theories or ideas should be received cum grano alis: and, until there is a large accumulation of facts, carefully obuntil ther and accurately recorded, it will be wise to consider this whole subject still sub judice.

\section{FACTS FOR NON-CONTAGIONISTS.}

Having already detailed* a class of facts, which appear to be corroborative of the doctrine of the contagiousness of to be corroborative it but fair to the supporters of an opposite opinion to record a few observations, which may be regarded by them in the light of counter-evidence. While, however, I admit that, viewed in particular lights, the undernoted facts may be accepted, to some extent, as evidence of the non-contagiousness of the disease, I hold them to be easily explicable in connexion, and to be perfectly consistent, viewed in certain other lights, with the adverse theory. The class of facts to which I allude consist chiefly of the following:

I. Immunity of persons engaged in experiments and investigations on the nature of cholera, and who are more or less constantly respiring the cholera poison in its worst forms.

II. Immunity of medical men in more or less intimate contact with the sick.

III. Immunity of the matrons, officers, and attendants of cholera hospitals.

Iv. Immunity of persons who have accidentally swallowed the evacuations of cholera patients.

v. Immunity of persons accidentally and unknowingly in contact with the sick.

vI. Immunity of particular members of a family, inmates of a house, or inhabitants of a street, town, or district; while other persons and places, less immediately in contact with the sick, or contiguity to infected localities, are attacked.

vir. The simultaneity instead of the succession of outbursts.

virr. The fact of cholera travelling against the wind, across the sea, overleaping whole tracts and countries, etc.

In illustration of the first class of cases, I may mention that when there were cholera cases in the City Cholera Hospital of Edinburgh, my time, daily, for some weeks, was to a great extent divided between

I. The vards, where my clinical duties in observing and reporting cases, in every stage of the disease, required me to come into very intimate contact with the sick. While using the stethoscope in sereral cases, with a view to the detection of latent or obscure pulmonary lesions, making thermometrical observations on the temperature of various parts of the body during the collapse and other stages, or testing the acidity or alkalinity of the sweat and saliva, I frequently inhaled the effluvia from the breath and sweat of living patients; and, while making galvanic experiments on the bodies for some hours after death, I had equal opportunities of inhaling the effluvia from the skin of cholera corpses.

II. The laboratory, a very small apartment, where I was engaged investigating the histology and chemistry of the evacuations, and the histology of the pathological lesions in cholera patients, and where the atmosphere was seldom free from cholera effuvia, arising from evaporating down evacuations in all stages of freshness and putridity, or from subjecting them to other chemical operations.

III. The experiment room, also a very small, damp, close apartment, where I had confined the animals that were the subjects of experiment on the transmissibility of cholera, where the atmosphere was contaminated by the effluvia arising both from the evacuations and viscera of cholera patients, supplied to the animals as food, and from the bodies and evacuations of the animals themselves, and which $I$ had occasion to enter sereral times daily, to watch the progress of the experiments: and less frequently

Iv. The post mortem theatre, where the pathology of fatal

- Associntion Jodrinal, Sept. 15th, 1854. cases was carefully examined, and where I wis axposed to the effluvia from the blood and viscers of dead patients.

I must, therefore, have been almost constantly respiring, in some shape, the effluvia from the skin, lungs, and erackations of living patients, and from the bodies and viscera of dead persons, as well as of the animals which died under experiment. Nloreover, my hands were occesionally cut and abraded by the manipulations in which I was engaged: still my health suffered in no way from this unusual amount of exposure. In connexion, it may be serviceable to mention, that I was in previously good health; that I endeavoured to maintain the healthy equilibrium, so far as my duties allowed, by regularity in diet, exercise, etc. ; that I do not appear to be predisposed to contagious disenses, never having been attacked, while engaged in hospital duties, by typhus, measles, or scarlatina, by dissecting-room fever or boils, or the numerous other ailments to which many medical students are so liable; that there were seldom more than two to four patients in hospital at the same time, and that there were periods during which we had no patients; that the hospital was commodious and well rentilated, and that mr sleeping apartment was separated considerably from the wards; that I seldom had laborious night duties, and that I had no out-of-door duties; and that, having at the time full faith in the Board of Health views on the non-contagiousness of the disease, $I$ was not in the least afraid, and, indeed, never calculated on the possibility, of being myself attacked. I am bound, howerer, to confess my belief that, had I been predisposed by fear, by bad health, by close confinement and laborious duties, by exposure to cold, by starvation, or by similar circumstances, either constitutional or external, the results might have been far different. Several continental experimenters, who have been similarly exposed to the cholera poison, have enjoyed an equal immunity.

The matron of the hospital, who was in almost constant attendance on the patients, and who had been greatly exposed to the disease in former and much more severe epidemics, was also exempt from any attack. But she also was in good health, and knew nothing of fear; her coustitution was, moreover, naturally very robust.

It is needless to multiply similar cases, or to illustrate the other classes of cases above mentioned, with the exception perhaps of class IV. Instances and illustrations must be familiar to every one conversant with the history of cholera, in this or previous epidemics.

At present, I can recall to memory only ohe instance of swallowing evacuations, in the case of a man, in a state of inebriety, who inadvertently swallowed half a tumblerful of the romit of a cholera patient without any subsequently bad effects.

But the key to the immunity in all the above, and in similar cases, I consider to be non-predisposition in the individuals. It is impossible to over-estimate the value of the subject of predisposition in the study of a question of such importance and difficulty as the contagiousness or non-contagiousness of cholera. We have only to look at the frightful ravages of this disease after the battle of Alma, to obtain a true estimate of the influence of predisposition on the mortality of cholera. In private letters, published in the public newspapers, from the survivors of that bloody fray, we are informed of many instances of healthy individuals, unaffected by cholera up to the moment when they suffer extreme exhaustion from the fatigues during or after the battle, who are thereby suddenly reduced to such a state of debility, that they fall easy victims to the fell destroyer.

The want of sufficient exposure to the cholera poison, and other circumstances, which I have already mentioned, must also be allowed some share in the production of exemption from cholera in certain indfviduals and localities.

The subject of the

TREATYERT OF CHOLEBA

has been recently brought so prominently into notice in the public journals, that it admits of very fer remarks from 
2. The mear fnet of the extrome contrariety of opinions - to thection of remedies, exhibited in the infinitude of Intwer from all parts of the country, which have been pubithed by the medical press during the present epidemic, is of itedf suficient to indicate the unsatisfactory footing on Which the subject still stands.

Romedies the most incongruous, diversified, and opposite in their actions, have been lauded as specifics ; treatment the most abeund, both in theory and practice, is daily had recourse to, dictated in some cases by the most wild and extravagant theories; in others by mistaken notions of pathology; and in others by blind empiricism, hap-hazard, or a reckless and aimless love of experiment. It is by no means casy to perceive how in one man's hands purgatives or sedatives, and in another's astringents and stimulants ; or how castor or croton oil, opium, strychnine, brandy, cajeput, peppermint, and a host of the most discondant remedies should be equally effectual in the cure of the same disease. There can be no better proof of the utter fallacy of the supposed value of all our vaunted specifics, than the simple fact that medicines possessed of every variety of action, physiological and therapeutic, on the human body, have in their turus enjoyed the enviable, but in all cases transient, reputation of being "Cures for Cholera". It is not by making the unfortunate victims of cholera the recipients, in turn, of all the articles of our materia medica, for the purpose of studying their random effects, that we can, $a$ priori, expect to arrive at valuable scientific truths rcgarding the laws of cholera; but by accurate labours with chemical, microscopical, meteorological and other instruments, by analytical experiments on man and animals, and subsequently by gynthetical investigations, whereby we may test and prove the truth of deductions and generalisations arrived at by the previous means of research. The medical journals teem with ephemeral and futile correspondence on the treatment of cholera ; and it cannot but be regarded as an unfavourable "sign of the times" regarding medical education in this country, when we find this strong tendency to empiricism on the part of the many triumphing over the rational application of analysis and synthesis, and all the lights of modern discoveries in science, which is confined to the few. My object is, therefore, not to add to the huge list of vaunted specifics or useful remedies, but to point out the superiority of leaving nature greatly to herself in the management of the disease, to indicate the advantages of passive over active treatment.

In connexion with the use and abuse of drugs in cholera, it is important to bear in mind especially the following points.

I. The tendency of the disease to run a regular course the chances of a spontaneous favourable termination; and the danger of confounding the natural tendencies to amelioration in the symptoms with the therapeutical effects of certain medicines.

II. The suspension of the function of absorption during certain stages of the disease, in consequence whereof medicines are expelled comparatively unaltered in composition or quantity, and must be inert.

Many of those who put implicit faith in particular drugs in the treatment of the disease, seem to be ignorant of the natural features of its various stages and phases under different circumstances; of their spontaneous tendency to recovery ; or of their mode of termination. Due allowance is not made for the great variability of symptoms according to peculiarities of age, sex, constitution, and predisposition, the type of epidemic, the customs of nations, the physical geography of a district, the sanitary condition of a locality, and similar circumstances. It is constantly stated as an indication of farourable progress under the use of a particular drug, that the stools gradually become dark, bilious, and foetid. I have already fully shown that this is the natural tendency of the disease, independent of, and eren in opposito, therapeutic means, and that, in some cases, the "ricewater" stools are in very small quantity, or may be altogether absent. Nor do I think the causes or modes of death in cholera hare attracted sufficient attention.

\section{It occure variously by}

I. Synoope, from sudden failure of the beart's action during some unusual exertion while in collapee, or under the influence of the warm bath; or from succuseion of the gystem in the course of conveyance from a private dwelling to a public hospital. I have known of death by syncopo in one case where an attack of cramp caused a sudden rising from her pillow; in another, where the patient, a collapse case, was gently raised while some bed clothes were being adjusted; in others, while collapse cases were being mored into or out of the bath.

II. Asthenia, during collapse, probably from the dynamic action of the cholera poison on the ganglionic nerve system. The majority of collapse cases appear to die in this way.

IIr. Asphyxia in later stages, from the accumulation of blood and serum in the lungs, especially posteriorly and inferiorly, from gradual accumulation of mucus in the bronchi, or from bronchitis, pneumonia, or other pulmonary complications. These chiefly occur in the typhoid stage, and are the results of the systemic debility.

Iv. Coma, during the typhoid stage, the result of the suspension of the renal function, or of uræmic poisoning of the blood.

The attributing to the curative effects of inert substances phenomena which are in reality natural to the spontaneous progress of the disease towards its termination, is doubtless the key to the great success of hosts of much lauded specifics.

Some experiments lately made in France by Duchaussoy* prove that during the algide stage of cholera absorption along the intestinal tract is greatly disturbed or altogether suspended, and that medicines given in this condition of the mucous membrane remain inert till reaction is established. He found, for instance, that belladonna, given during collapse, had no effect on the pupil, which, however, gradually became manifested as reaction set in. As a consequence, it follows that medicines given during this stage are not only inert and useless so far as the treatment of the disease is concerned, but that they accumulate, and during reaction and the subsequent stages must in many cases be productive of serious mischief. If these observations be correct, and I believe they have been elsewhere corroborated, we are at once in a position to see the inutility of drugs of any kind during collapse, and the probability that the untoward symptoms of subsequent stages are frequently the result of the medicine thus imprudently and unnecessarily given. This will account for the fact that the upholders of the calomel practice find no trace of mercury in the system after repeated and large doses of that drug, which has simply passed through the intestines and been expelled in the stools, where it has been frequently detected in considerable quantities, unchanged, and unabsorbed.

Ny own experience in the treatment of cholera consists chiefly of the following two classes of cases :

I. Where the management of the case was left chiefly to nature, the spontaneous progress towards recovery being assisted, and the tendency to death in cach particular case being obviated by simple measurc., Ich as the maintenance of animal heat by copious warm bathing, and a plentiful supply of warm and nutritious food. IIere drugs were thrown aside, in order that the unmodified and uncomplicated features of the disease might be dispassionately studied, wherehy an estimate might be formed in every individual case of the relative chances of recovery or death, and a plan of rational treatment laid down to assist in the one case, and obviate in the other, the modes in which these severally occur.

Ir. Where several or all of the remedies usually recommended in cholera were experimentally exhibited. In many of the fatal cases, the patients had swallowed successively large quantities of one or more of the so-called specifics,never in any case with a result satisfactory to myself.

Contrasting these two classes of cases, about an equal

* On the Absorption of Nedicines in Cholera-Gazette Hebdomadaire de Méd. et de Chirurgie, Sept. sth, lisut. 
number of recoveries and deaths happoned in each, which enficiently proved to my mind the inefficacy of the drugs in the latter class. But these are not only useless, for the reasons just explained, but they are productive of effects which are fertile sources of difficulty and doubt as to prowhich are fertile sources of difficulty and diagnosis, by masking or modifying the natural gnosis and of the disease. By this means, the treatment of the later stages of cholera, especially the typhoid fever, is materially interfered with, and a fatal issue may frequently happen from unistakes thus induced. My own quently happen from mistakes on practice and guided by theoretical views, are strongly in favour of the vis medicatrix natura, assisted by the simplest and least objectionable means already mentioned.

I beliere by far the most important point in the treatment of cholera is the securing of efficient attendants upon whom you can depend to carry out faithfully the minutio of your orders. A careful, conscientious, kind nurse is here of paramount importance. Supply nurses thoroughly educated to their duty and who will unshrinkingly do it, not from mere mercenary motives, but from a spirit of Christian benerolence, and I believe drugging in cholera to a great extent useless : but no amount or kind of medicine will form an efficient substitute. My faith in favour of lcaving the treatment of cholera in the hands of nature and careful nurses is abundantly corroborated by the experience of the profession in former epidemics. It is sufficiently notorious profin severe visitations of the disease in different localities in Scotland in former years, when many cases were totally left to themselves in consequence of desertion by friends, or the inability of the medical officers to pay them any attention, these constituted the first and most favourable recoveries; and the mortality was seldom or never found greater in cases thus abandoned than in those subjected to the most active and careful treatment by the most experienced members of the profession. Besides, many cases, carefully attended by medical men, dic from negligence or inattention on the part of those who act as nurses or attendinattention on tallent movement has lately taken place in Zondon to educate a staff of women, to be drawn from our unions or poorhouses, as nurses to the labouring classes, especially during periods of epidemic sickness. I would respectfully call the attention of civic and parochial authorities to the extreme importance of a staff of educated cholera nurses for the supply of cholera hospitals and private houses, especially in country districts where the fatal effects of panic render their assistance more necessary than in towns. There is an abundant materiel in all our workhouses, houses of refuge, and similar charities for the reception of indigent women; all that they require are systematic training and constant supervision. Every parochial inspector knows full well the dreadful effects of panic on the inhabitants of a small country town, or village, on the first outburst of cholera, especially if it be known to be virulent in the vicinity, and the extreme difficulty and annoyance he has experienced in procuring professional assistance of any kind. By the means which I have just hinted, he has it in his power easily to provide for future contingencies; but the subject is one of such importance that it merits the careful attention of the Board of Health and Government.

The only remedial means on which I will offer any remarks are the warm bath and chloroform : this I do from the belief that both are liable to great abuse in the treatment of cholera, and being so, that their indiscriminate use is fraught with great danger. I could say much of the effects, physiological and therapeutical, of various remedies which I have seen tried, but as these do not bear essentially on the curability or rational treatment of the disease, I will refrain, at present, from doing so.

As a general rule, it is adrisable, immediately on the admission of pauper patients into cholera hospitals, to plunge them into a warm bath, of temperature about $90^{\circ}$ to $98^{\circ}$. This class of patients consists chiefly of the offscourings of society, half clad, half fed, squalid wretches, living in filth and misery, and exposed to the vicissitudes of the weather. The bath serves the double purpose of ablution and re- storation or elevation of the animal heat. But, under cone. tain circumstances, its use is attended with great danger: the sedative effect of the heat is frequently fatal. Where it has boen twice or more frequently had recourse to in the me patient, I have known the first application beneficial, while the subsequent ones were as decidedly deleterions. After a prolonged immersion of fiftoen to thirty minutes, the pulse was usually found to increase in fulness and rapidity, or to appear, if formerly absent; and the skin acquired a genial and comfortable glow of heat. In other cases of confirmed and advanced collapse, even longer immersion was not followed by any appearance or increase of the pulse, though a temporary heat was communicated to the body. In the former case, it generally happened that, after a few minutes' immersion, the patient expressed a feeling of relief and comfort, until perhaps a sense of faintness or sickness was induced, indicating that the application of the heat was being carried too far, and might be attended with danger from death by syncope: in the latter, the patient usually complained of the scalding heat, though the temperature was usually below $98^{\circ}$; so great was the difference between the real temperature of the water and the perceptions of the patient : and he made violent struggles to escape from the bath. In such circumstances, prolonged immersion was fraught with peculiar and sometimes immediate danger; and death might happen during removal to bed. One useful effect of the systemic revulsion produced by the heat was the partial cessation of the diarrhoea: in some cases, astringents were found wholly unnecessary after the bath. It was also of great importance as a diuretic ; indeed, in this respect, the hip-bath was superior to colchicum, spiritûs æotheris nitrosi, squill, et hoc genus: omne. It was not, however, efficient in preventing cramps, or in mitigating the pain thereby produced, though it appeared to lessen the tendency thereto pro tempore. I repeat, therefore, that I believe the application of moist heat. by means of the warm bath in cholera requires great caution.

When chloroform was given by inhalation, to mitigate or obviate cramps, it appeared to me not only useless, but. highly dangerous. Insensibility being produced, the sensation of pain was necessarily abolished for the time being; but the spasms of the muscles of the leg and foot were in nowise controlled or prevented. In one case, where, however, the patient was not fully narcotised, a condition of slight delirium, marked by low muttering, was induced, which passed into a comatose state, attended by stertorous: breathing. He gradually and effectually recorered from the effects of the chloroform, though, unfortunately, not from the effects of the cholera, as he died shortly afterwards.

However great may be the variety of opinion regarding. the treatment of cholera, there ought to be little diversity regarding its prevention on the great scale by hygienic means. I shall, therefore, for the present, conclude my "Clinical Notes on Cholera" by a few remarks on

SANITART REGULATIO:S IN RELATION TO THE PKEVENTION, MITIGATION, AND ARRESTMENT OP CHOLERA.

It is almost unnecessary here to recapitulate the powerful influence which the various infringements of the sanitary laws, so common in the more populous localities of our larger towns, have on the origin and spread of cholera, augmenting its virulence, and multiplying the foci of contagion. So long as we permit in our towns narrow, dark, crowded alleys, and tall, closely-packed, half-ruinous houses, entered by dismal filthy stairs, and containing rooms which more resemble pigs' litters than the habitations of human beings ; so long as we allow our poorer population to remain cooped up in these miserable abodes, in a condition infinitely worse than that of our beasts of burden; so long as we have huge accumulations of offal and rubbish in the centre of some of our most densely peopled localities; defective drainage and water supply; stagnant gutters and choked sewers; deficiency of public necessaries and urinaries; colonies of pigstyes and byres; knackers' yards and 


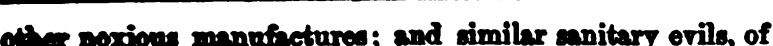
Wich for town are free, - Te cannot expect exemption from typhys and cholers, and their congeners. It is in vain that civic and parochial authorities are informed that the proper opportunity for fortifying the localities under their juriediction against the inroads of disease, by carrying out annitary measures dictated by modern discoveries in social and sanitary economy, as well as in the sciences more or loss intimately bearing thereon, is during freedom from epidemic disease, during periods of health: and it is equally vain that the medical profession constantly forewarn them that the fell destroyer is about to visit their towns or villages. It is not till the epidemic actually bursts forth that active measures to protect themselves, and the community under their charge, are taken. Then it is too late; the steps taken are not only unattended by the wished for resultthe mitigation or arrest of the disease-but they are not unfrequently productive of effects the most unfortunate, and the most opposite of those intended. The efforts even then made are very superficial: alleys are whitewashed; drains flushed; dungheaps remored; cesspools opened up and cleaned; pigstyes and noxious manufactures are broken up or removed; and marshes are drained: and all this usually after the disease has giren unequirocal evidences of its presence in the place. The inutility of these proceedings I may illustrate by the fact that, in a number of cases of cholera, in which I made this the subject of special inquiry, I found that the exterior of the closes or houses had been whitewashed and the gutters flushed several days or weeks prior to their seizure. But, while in the majority of cases useless, these sanitary measures cannot be said to be harmless; for I have known several cases of fatal cholera apparently induced by the means taken to clean houses in which members of the family or neighbours had previously been attacked. Let us, for instance, suppose such cascs as the following-very simple forms of the baneful effects in question.

I. Three members of a family are seized with cholera; one dies; the remainder are removed, on the funeral day of the first, to a cholera hospital. The cases are reported, in the usual way, to the authorities, who send an inspector to examine the house in which they occurred, and to report. He describes it as small, dark, confined, and filthy. Without reference to the comfort of the remanent members of the family group-without making temporary provision for their health and comfort, by removing them to an observation ward or house of refuge-an order is given to whitewash and clean the house; doors and windows are thrown widely open; the whitewashers do their duty, irrespective of furniture, and leare the walls and floor wet. This is done independently of, and probably in opposition to, the wishes of the occupants of the house, who must submit. The doors and windows are ordered to be left open during the night, for the purpose of facilitating the drying of the paint, which apparently is supposed to be endowed with powerful protective or healing virtues. The season is winter; the air is damp; the cold is piercing; perchance snow or sleet are drifting in through the open windows: the atmosphere of the room consists of a series of cold currents or draughts. Another member of the same family, a mother, in a state of intense anguish at the death of one and the illness of others of those nearest and dearest to her; fatigued with the labour of attending on their sickbeds; annoyed at the ruthless intrusion of the whitewashers, - finds herself without a bed, from the confusion into which her furniture has been thrown, or perhaps from its having been burned by the authorities, to prevent the risk of further infection: she stretches herself on two chairs before the fire. She has been in good physical health up to this fatal night. Cold, shivering, hungry, she passes the night miserably before the dying embers, and next morning she is seized with premonitory symptoms, which rapidly pass into the worst form of the disease; and she too is removed to the cholera hospital. Some kind Samaritans among her neighbours take charge of her remaining children; the authorities hear of another case in this unfor- tunate house; some of the worst furniture is burned; and the house is shut up.

II. $\Lambda$ husband is seized, sent immediately into a cholera hospital, and dies of cholera. The case is again reported; the house is officially examined; it is whitewashed; and, as in the former case, at night the doors and windows are thrown open, and the walls and floors are left wet. His wife, up to this period in robust health, passes the night in the house; catches cold; is seized with the premonitory symptoms, which rapidly run on to collapse; and she dies before her husband.

Surely it is a legitimate deduction in these two cases to consider the exposure and other circumstances resulting from the steps taken by the authorities to clean the respective houses, as powerful predisposing causes of the disease. It is certainly possible, or even probable, that, independent of such exposure, these persons would have been attacked: but the chances are equally that they would not. In either case it cannot be denied, that the effects of the intended sanitary measures must have been the reverse of safe or salutary. Moreover, if we reflect for a moment on the consequences likely to follow,-during periods of nstional health, - from setting free the most noxious effluvia in populous localities; by stirring up dungheaps, accumulations of night soil, choked cesspools and sewers, in the process of removal or cleaning; or of opening old drains or privies, or cutting drains in malarious marshes, we shall be in a condition to appreciate the want of wisdom which dictates such proceedings during epidemics of cholera or typhus. The true remedy consists in preventing the accumulation of such sanitary nuisances, not in removing them after disease has indicated its presence; instead of meeting the intended ends, such measures only add increased fuel to the fire already kindled.

Before we can expect to eradicate contagious disease from the haunts of filth and miscry in our large towns, we must put our shoulders to the wheel of several reforms, which are neither few nor unimportant. It is not by whitewashing a few stone walls, flushing a few gutters or stagnant streams, or removing a few depôts of manure or filth, that we can expect to make rapid or satisfactory strides in the right direction. The initiative must be the education of our lower classes; the gradual elevation of their social condition; the improvement of their dwellings, and the construction of our towns on principles having more regard to the health and comfort of the mass of the people. We must educate the working classes to appreciate the full value of the laws of health, and the consequences of their infringement; we must enable them to understand the importance of cleanliness, temperance, and other cardinal virtues; at the same time we nust supply them, or enable them to supply themselves, with houses which are commodious, while they are cheap, and well situated with regard to ventilation, light, dryness, water-supply, etc. In a word, our whole sanitary and social economy requires revision ere the dregs of our population are placed in a position to withstand the inroads of epidemic disease. This is, at present, a reform "to dream of, not to see"; but, unti? it is consummated, we must be content to find cholera, like typhus, settling down familiarly : mong us-becoming endemic. While we confidently hope that the good time for such a reform will eventually arrive, we believe, in the meanwhile, that civic and parochial authorities would do well to consider carefully the following points :-

I. The substitution of permanent or constant for temporary or occasional sanitary regulations.

II. Improvement of the dwellings of the poor, by thinning of the inhabitants, and removing them from the crowded centres to the suburbs of towns.

III. The destruction of close, crowded, narrow allers; and the substitution of commodious open streets.

Iv. The formation of proper sewage.

v. Plentiful supply of water, of good quality.

vi. Formation of public necessaries and urinaries in large towns.

vir. Intimate supervision of the divellings of the poor. 


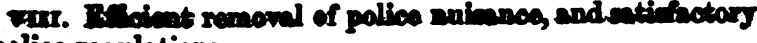
police regulations.

Ix. Bducation of cholere nunes, to be drafted from woxkhouses and public charities.

x. House to house visitation during epidemic cholera.

II. Frection of the cholern hospitals formerly allinded to*

Yurray's Royal Asylum, Perth, Norember 185t.

\section{CASE OF FRACTURE OF THE BASE OF THE SKULL: RECOVERY.}

By GEORGE BIDIE, M.B. and L.R.C.S.E.

Or Sunday, the 21 st of May, while visiting a patient in the country, I was hastily summoned to see a lad in the fohing village of Findochty, who had met with a serious accident. On my arrival, I learned that the sufferer, a young fisherman, aged 16 years, had been reclining upon the brink of a crag in the neighbourhood; and that, on getting up "to stretch himself", he had lost his equilibrium, and fallen down a height of from twenty to thirty feet. The head had first come in contact with the ground, and there was a deep ragged scalp wound stretching from right to left across the occipital protuberance. This gap had bled profusely; and on washing away the clots that hung in the hair, I discovered a copious hæmorrhage welling up from the left ear. The discovery of this hrmorshage, in conjunction with other signs indicative of serious mischief, led me to suspect fracture of the base of the skull-the fracture by contre-coup. To remove the more alarming symptoms of collapse was now my care-the pulse being very feeble and intermittent, and the heat of the body considerably below the natural temperature. In - little, frequent vomiting ensued; and I was gratified by seeing at any rate a partial recovery established. By this time, the bleeding from the ear had completely soaked the pillow. Taking, therefore, into account the aggregate loss of blood, I was led to consider further depletion unnecessary. No fracture could be detected at the site of the scalp wound, and the other parts of the body amenable to an outward examination seemed quite intact. I now cut the hair as close as possible, approximated the edges of the wound with strips of adhesire plaster, and had the head elevated upon pillows, with orders that it should be kept constantly covered with cloths wrung out of cold water. Before leaving for the night, I administered twelve grains of calomel rubbed up, as the power of deglutition was but feeble, in a pill of fresh butter.

Monday, May 22nd, 6 o'clock A.M. During the night, my patient had relapsed into a state of insensibility nearly as profound as the primary. The skin was hot and perspiring; the pulse slow, but rising upon any attempt being made to arouse him; the breathing was slow, deep, and somewhat stertorous; the bowels had been unmoved. One drop of croton oil was put upon the tongue, and an enema of turpentine ordered. The enema was directed to be repeated at intervals. A thin watery discharge had taken the place of the hæmorrhage from the ear.

7 o'clock P.x. The second enema had been administered about 12 o'clock at noon, and brought away large quantities of freal matter. Since then, the patient had twice intimated, by intelligible signs, a wish to have the chamber utensil when the calls of nature were urgent. Any attempt, however, to arouse him from the lethargy which still continued, merely tlicited a surly "be quiet", or a wish to be let alone. The pulse was still slow; the skin cooler; and the breathing freer.

May 23rd, 12 o'clock at noon. My friend, Dr. Gerrard of Buckie, kindly accompanied me during this visit, and formed the same opinion as myself regarding the nature of the injury and imminent danger of the patient. The stupor was still persistent; the pulse slow; the skin hot. He had spent a restless night. We ordered twelve

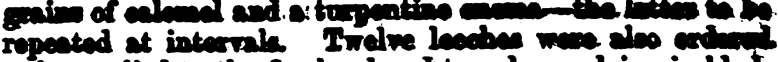
to bo applied to the forehead and temples, and ice in blact ders kept conntently upan the hoad

May 24th, 6 o'clock 4.X. The stuper was leas maked; the pulse had improred; and the borrell had been morect freely more than once. I ordened twelve powders, contrining in all twenty-four grains of calomel. Three of thees. powders were to be administered drily.

8 o'clock erening. He was ctill improving aince the morning.

From this date the pationt gradually progressed towands necovery, the treatment, with slight variations, being merely a repetition of the foregoing. The scalp wound healed kindly. During recovery he exhibited great wealmeese in the lower extremities, staggering like a paralytic on being aasisted to the erect posture. By the l0th of June, he could with a little assistance walk from his bed to a chair. For a time, however, after regaining the power of speech, his memory was completely upset. Iike some of the Athenians, who recovered from the great plague, mentioned by Thucydides, he actually could not at one period recallect his own name, or that of a single friend. The intelligent look, however, that his features displayed upon his being asked the name of any friend, seemed to indicate the power of recognition, although memory refused to supply the correct name, and furnished instead a nomenclature the most fantastic and inexplicable. His mother was frequently called "the tongs", "the book", etc.; and hardly ever by any chance did she twice enjoy the same appellative. Repeated blisters and a seton in the neck, along with the cautious use of mercury, shortly began, however, to effect a change; and by the middle of July, he was in perfect possession of all his faculties, physical and mental.

Cullen, December 4th, 1854.

\section{$\checkmark$ \\ CASE OF SUDDEN DEATH DURING THE INHALATION OF THE VAPOUR OF CHLOROFORM.}

By JOHN BIRKETT, Esq., Surgeon to Guy's Hospital.

HANNAH Brooks, aged 56 years, was admitted into Guy's Hospital, under my care, Nov. 15th, 1854. She said she was in good health; but she had a most cachectic appearance, was edentulous, and appeared quite ten years older than the age she represented herself to have reached. She lived in Southwark, and obtained a somewhat precarious livelihood by washing. She had had chronic ulceration of both legs the last two years, the ulceration nearly surrounding the leg, at about the junction of the middle and lower third. Two months before admission into Guy's, the ulcer on the left leg assumed a sprouting, fungating aspect, became very painful, and, during the last month, it bled frequently and profusely. In the left groin there was an enlarged gland. Various means having been ineffectually employed to destroy the cancer, amputation of the limb below the knee was proposed as the only palliative measure, and was agreed to. She drank some wine and water a short time before the administration of the chloroform. Her mind was calm and resigned, and there was no apparent depression produced by the contemplation of the operation. On Monday, the 4th of December, she seemed to be suffering from a trifling febrile attack, and said she thought she had taken cold. I told her that, if this condition existed upon the following day, we must postpone the operation. I gave her an ounce of mixture of acetate of ammonia, with fifteen minims of tincture of hyoscyamus, three times daily. On Tuesday, the 5th, she expressed herself as feeling quite well; the febrile attack had passed away; the skin, the temperature of which wes higher than the healthy standard the day before, was natural; and her tongue was slightly furred - a condition of this organ which had existed during the whole time of my observation of the case. A few minutes after 1 P.X., she was carried into the operating 